\title{
La concentration spatiale des activités productives au Brésil
}

\author{
Jandir Ferrera de Lima, Ph.D. \\ Université Estadual do Oeste do Paranà
}

\section{INTRODUCTION}

\section{La concentration, comme la polarisation, est le résultat de l'interaction entre les coûts réduits de transport et de relations interindustrielles de coopération et la concurrence dans des régions spécifiques. Pour cette raison, les régions périphériques allient coûts expressifs de transport avec une relation de dépendance dans les activités de transformation et de services.}

L'objectif de cet article est de discuter de la concentration spatiale des activités productives au Brésil. Il y a trois aspects théoriques qui expliquent le mouvement de l'activité productive dans l'espace. Le premier est l'intervention de l'État dans l'économie, qui, dans le cas du Brésil, est devenu l'appui du processus d'industrialisation, condition pour l'unification de l'espace économique. Le deuxième se réfère au rôle des ressources naturelles, historiquement la principale cause de l'ouverture de frontières économiques. Le troisième seraient les facteurs spécifiquement spatiaux, en particulier la dispersion des ressources naturelles qui influencent la diversification des activités urbanoindustrielles.

Sur cet aspect, la concentration, comme la polarisation, est le résultat de l'interaction entre les coûts réduits de transport et de relations interindustrielles de coopération et la concurrence dans des régions spécifiques. Pour cette raison, les régions périphériques allient coûts expressifs de transport avec une relation de dépendance dans les activités de transformation et de services. Cela fait que les régions périphériques ont un coût de production et de distribution plus élevé allié à des problèmes de retours à l'échelle. Les coûts réduits de production associés aux retours à l'échelle et des coûts de services peu significatifs engendrent des tendances à la concentration géographique des agents économiques, conséquemment dans le niveau de localisation des activités productives et dans l'organisation spatiale de l'économie'

Dans une ligne d'analyse, la dispersion des activités productives exige l'implantation de politiques d'intégration régionale et de création d'une identité collective. Cependant, son implantation trouve les intérêts politiques locaux comme principaux obstacles, puisque l'intégration et l'amélioration des conditions d'éducation et de qualité de vie impliquent la création de conscience politique et un changement au centre du pouvoir des régions. En plus, le changement au centre du pouvoir est directement lié à l'organisation politique des régions. Ce qui peut être un obstacle dans certains de ces processus et un élément balise dans d'autres. Finalement, la question politique change selon les caractéristiques de chaque région et des directives nécessaires pour implanter l'intégration. Le processus d'intégration régionale et la formation d'une identité propre impliquent des réformes, lesquelles induisent la négociation avec les groupes politiques locaux et les représentants de la société civile. Elles impliquent autant les questions liées au transfert de revenu et de richesse que celles liées à l'organisation sociale de la région. La forme selon laquelle ces questions vont être mises en œuvre dépend de la cohésion des intérêts de ces groupes $^{2}$.

Le processus d'intégration régionale et la formation d'une identité propre impliquent des réformes, lesquelles induisent la négociation avec les groupes politiques locaux et les représentants de la société civile. 


\section{LA DÉCONCENTRATION ET LA «RE-CONCENTRATION» DE L'INDUSTRIE AU BRÉSIL AU XX ${ }^{\mathrm{e}}$ SIÈCLE}

Entre 1900 et 1960, la tendance de l'économie brésilienne était de concentrer la production industrielle dans la région Sud-Est du pays (figure 1). L'occupation spatiale de cette région a commencé au XVIII ${ }^{\mathrm{e}}$ siècle. Ainsi, à la fin du $\mathrm{XIX}^{\mathrm{e}}$ siècle, les régions situées à l'intérieur des États de São Paulo, Rio de Janeiro, Minas Gerais et Espíritu Santo ont eu un peuplement considérable en relation aux Régions Sud, Centre-Ouest et Nord. Ce peuplement a été stimulé pour que la production de café soit dynamisée, fait consolidé avec l'arrivée des immigrants étrangers. La croissance et la capitalisation de la production de café, l'approfondissement de la division sociale du travail, la fin de l'esclavage, la croissance de la population et des moyens de transport afin de transvaser la production agricole de l'intérieur des États de la Région Sud-Est, seront des éléments importants pour créer une demande interne chaque fois plus grande.

Pour cette raison, le processus d'industrialisation du Sud-Est brésilien a eu deux types de stimulus : la production de biens d'exportation et la distribution d'une partie du capital du secteur d'exportation sous la forme d'investissement et de salaires. Le progrès du Sud-Est, dans l'organisation spatiale de la production et dans la formation d'un marché interne, a permis qu'il arrive en première position en matière de croissance économique de la population du Brésil au $\mathrm{XX}^{\mathrm{e}}$ siècle. Différemment du Sud-Est, les régions Nord, Sud et Centre-Ouest auront leur peuplement effectif et l'avancée de l'occupation spatiale seulement à partir de la seconde moitié du $\mathrm{XX}^{\mathrm{e}}$ siècle.

Figure 1 - Division territoriale du Brésil : États et régions à la fin du $\mathrm{XX}^{\mathrm{e}}$ siècle

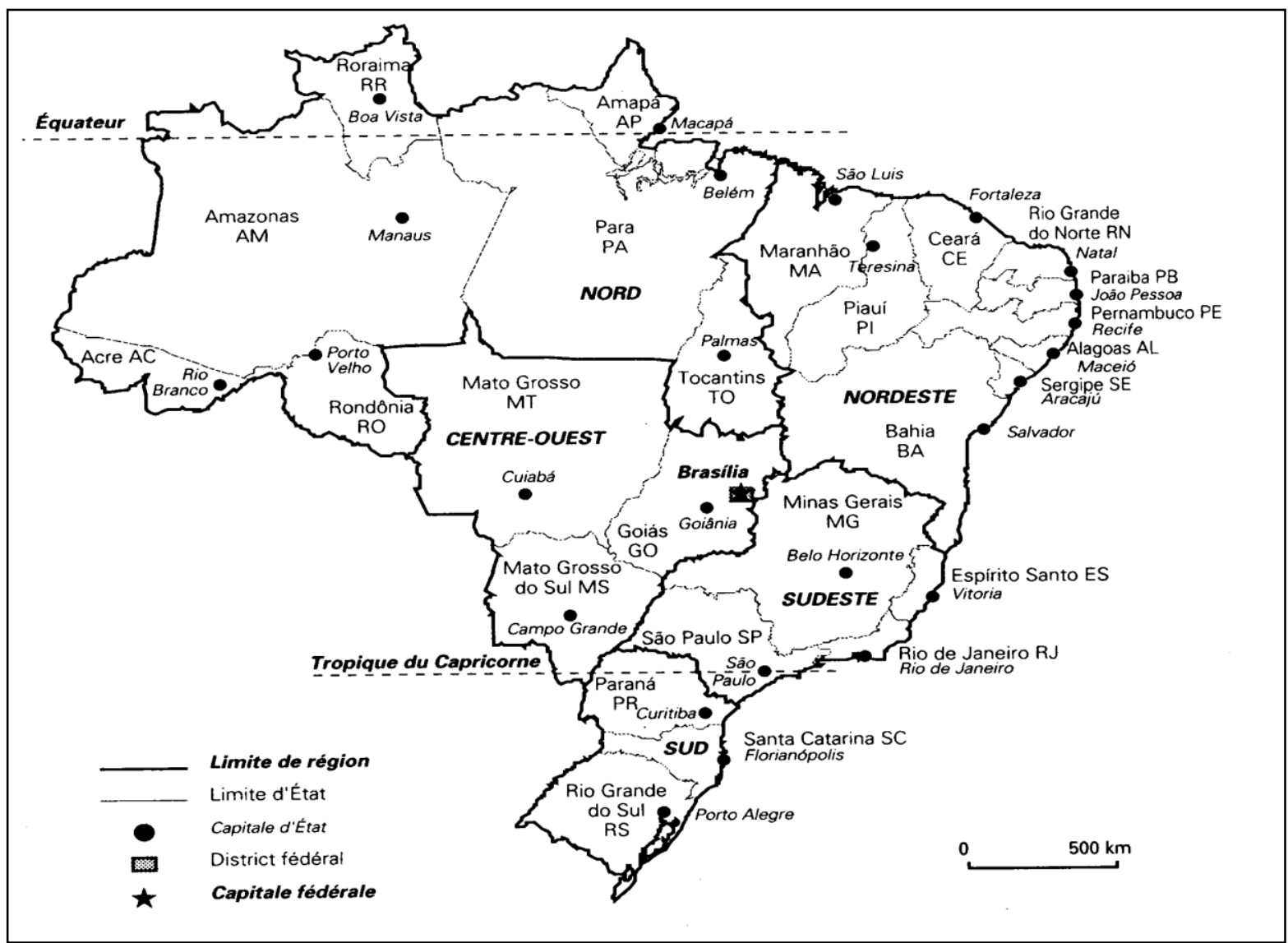

Source : Théry, H. (2000). « Le continent Brésil », Hérodote : revue de géographie et de géopolitique, $\mathrm{n}^{\circ}$ 98, p.9-28. 
Après 1970, un mouvement inverse commence à se produire dans l'économie brésilienne : le retour de la polarisation du Sud-Est en direction du Sud. Ce mouvement n'arrive pas par hasard. Le retour de la polarisation va se produire dans trois contextes ${ }^{3}$.

D'abord, la nécessité d'expansion des entreprises de transformation et de services du Sud-Est vers les nouveaux espaces du pays cherche à conquérir de nouveaux marchés et à montrer sa présence sur les nouveaux fronts de colonisation et d'occupation. D'un autre côté, le développement de nouvelles technologies de communication, l'avancée d'infrastructures de transport, la croissance démographique et les améliorations dans la productivité agro-pastorale laisseront l'espace de l'Ouest et du Sud brésilien plus attirant pour les nouveaux investissements. Ensuite, les politiques gouvernementales dont l'objectif étaient de produire des externalités positives dans les régions périphériques. Dans ce cas, dans les années 1970, les politiques de redistribution et d'organisation du territoire fomentées par le gouvernement fédéral ont stimulé un changement dans les tendances de la concentration. Pendant cette période, les grands réseaux de transport et de communication ont été planifiés et exécutés, couvrant une bonne partie du territoire.

\section{Le développement de nouvelles technologies de communication, l'avancée \\ d'infrastructures de transport, la croissance \\ démographique et les améliorations dans la productivité agro-pastorale laisseront \\ l'espace de l'Ouest et du Sud brésilien plus attirant pour les nouveaux investissements.}

De la même forme, un système d'avantages fiscaux et financiers pour les projets d'investissements industriels, agricoles et d'extraction minérale dans les régions périphé-riques a été développé. De plus, de grandes centrales hydroélectriques au Sud et au Nord du pays ont été implantées. Enfin, dans les années 1980 et 2000, il y a eu l'effet des économies d'agglomération, engendrées par les zones métropolitaines de São Paulo et de Rio de Janeiro. Ces économies d'agglomération ont eu comme conséquence l'augmentation des coûts des terrains, la pollution et la congestion urbaine, qui stimulera aussi la déconcentration vers le Sud du Brésil.

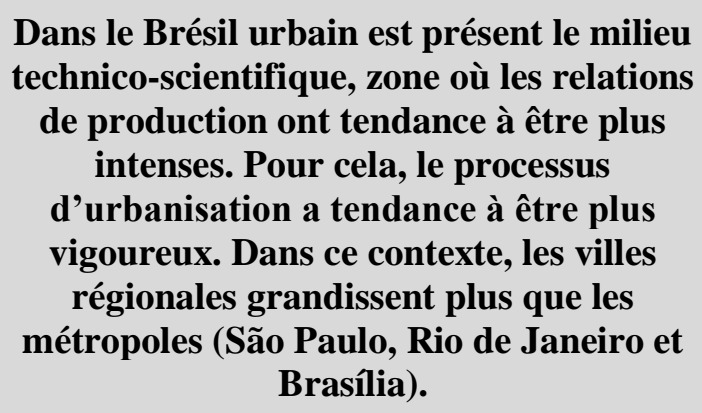

Un autre fait important qui s'est vérifié au Brésil est la tendance à l'urbanisation chaque fois plus accentuée. Il y aurait une dichotomie entre le Brésil urbain et le Brésil agricole. Dans le Brésil urbain est présent le milieu technicoscientifique, zone où les relations de production ont tendance à être plus intenses. Pour cela, le processus d'urbanisation a tendance à être plus vigoureux.

Dans ce contexte, les villes régionales grandissent plus que les métropoles (São Paulo, Rio de Janeiro et Brasília). Les villes moyennes ou intermédiaires présentent chaque fois plus d'attrait populationniste. Ces villes moyennes exigeront chaque fois plus de travail qualifié, alors que les métropoles, par leurs propres caractéristiques, vont accueillir des populations pauvres et désemparées. Même la migration qui s'est faite vers les dernières frontières agricoles a été extrêmement «urbaine", ceci étant principalement dû à la taille de l'urbanisation, qui ne peut pas être absorbée par les projets de colonisations.

Pour favoriser ce processus, il serait nécessaire d'améliorer les conditions de transport et de lien avec le centre dynamique de l'économie. L'agriculture de la région présente des signaux d'épuisement et affronte la concurrence des produits venant d'autres pays, principalement ceux originaires du Marché Commun du Sud (Mercosul), ce qui diminue la croissance du secteur agroindustriel (processus accéléré par le déplacement de la frontière agricole vers d'autres régions du pays). 


\section{NOUVELLES TENDANCES DE LA DISPERSION SPATIALE DES ACTIVITÉS PRODUCTIVES AU BRÉSIL}

Même si certaines tendances à la déconcentration des activités productives sont claires, on constate également que le développement économique brésilien est encore très irrégulier. Il existe dans l'économie un centre dynamique São Paulo - avec des relations de production dignes d'un pays du premier monde, qui investit en technologie de pointe et en compétitivité, et des régions qui vivent dans une situation d'extrême pauvreté et où les relations de productions sont similaires à celles du début du siècle.

Il y a des signaux selon lesquels le développement commence à se disséminer par l'économie. Les entreprises cherchent à fuir des externalités négatives d'agglomération, et c'est cette «fuite» qui sera bénéfique aux autres régions. Cependant, ceci est un processus très lent, avec des flux et des reflux, et qui sous le commandement exclusif des lois de marché deviendra encore plus lent. Il y a une tendance chaque fois plus importante pour la différenciation et la complexification, autrement dit, les villes et les régions seraient chaque fois plus différentes les unes des autres. Il se produirait également un processus de déconcentration géographique de la production, même étant un processus, d'une certaine forme très subtil, et qui n'affecte pas la domination économique de São Paulo sur le restant de l'économie. Pour cela, il faut planifier avec le gouvernement des mesures pour «distribuer» le développement économique.

Ce qui doit se produire, c'est que le processus de réorganisation brésilienne se ferait dans une région ample, ayant São Paulo comme centre et avançant vers plusieurs directions. La «forme spatiale » assumée par la société brésilienne a construit un centre dynamique (zone métropolitaine de São Paulo) et plusieurs types de périphérie aux alentours.

La première serait une amplification du centre São Paulo et s'étendrait aux villes de Rio de Janeiro et Belo Horizonte, en incluant également la zone agricole de grande productivité constituée par l'intérieur de São Paulo, le nord de l'État du Paraná, le triangle Mineiro, le Sud de Minas Gerais et les parcelles de Goias et du Mato Grosso do Sul.

La deuxième périphérie se constituerait dans les États du Paraná, Santa Catarina et Rio Grande do Sul, Mato Grosso, Goiás, sud-est du Pará, Rondônia et Acre, qui sont liés au centre à travers l'infrastructure des communications et de complémentarité d'activités économiques. Et en dernier, la Région Nordeste, avec une importante densité populationnelle, une faible productivité dans l'agriculture et des relations de compétitivité avec un centre dynamique, perdant une position relative au fil du temps.

À travers l'analyse du processus d'intégration économique, du mouvement de l'activité productive à l'intérieur de l'économie, la nécessité de planification du développement régional devient évidente, encore plus lorsque l'on perçoit que des populations brésiliennes sont encore obligées de déménager de leurs régions d'origine à la recherche de meilleures conditions de vie. Actuellement, le plus important est de diminuer les disparités régionales et de reprendre la croissance économique de forme sustentable. Le gouvernement brésilien a un rôle important, pour éliminer l'existence des «deux Brésils »: un riche, développé, compétitif, instruit et en bonne santé; l'autre pauvre, retardé, analphabète et sous-alimenté.

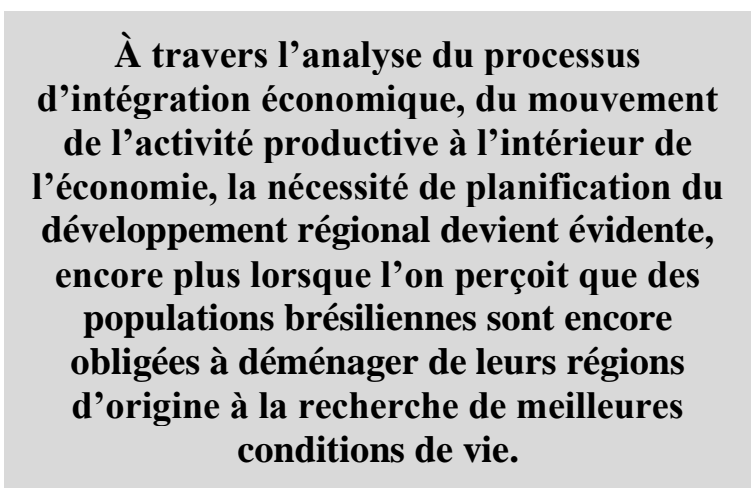




\section{CONCLUSION}

\section{La restructuration a été plus significative entre 1985 et 1995 . Pendant cette période, l'économie du Brésil est passée par de profondes transformations \\ macroéconomiques, parmi lesquelles on \\ compte la stabilisation des prix, l'ouverture commerciale, les changements dans la politique cambiste et l'entrée d'investissements externes directs.}

Cet article a examiné les disparités régionales au Brésil et le mécanisme par lequel ce processus se structure. Ainsi, on note qu'au Brésil, une politique de développement régional doit considérer deux éléments.

Sur le plan des déséquilibres sectoriels et au moment où se créent des branches industrielles tirées par une industrie «motrice ou pôle», s'impose un processus de substitution d'importations des matières premières sollicitées par cette industrie. Avec ceci, pour maintenir le rythme de croissance, on doit maintenir le processus de substitution d'importations dans d'autres secteurs. Il convient alors d'identifier les secteurs qui aujourd'hui sont substitutifs au nouvel arrangement économique du Brésil. Audelà bien sûr, d'investissements en gains de productivité et dans le surpassement d'étranglements sectoriels.

Sur le plan du niveau d'absorption de maind'œuvre, au moment où l'industrie «motrice» croît et absorbe une technologie de pointe, visant des gains d'échelle et une amélioration dans sa compétitivité, il y a une grande possibilité de diminuer le rythme d'emploi de la main-d'œuvre. Allié à cela, le haut niveau d'urbanisation cause une dimension de marché incompatible avec le rythme de croissance de la capacité productive qui s'est installé pendant cette période. Pour surmonter le processus de décélération dans le rythme de croissance et gérer de nouveaux enchaînements productifs de forme dynamique, on suggère l'accroissement de la base d'exportation des régions brésiliennes, de forme à absorber la maind'œuvre excédante, à recomposer la capacité installée des entreprises locales à partir de la demande des autres régions. Pour rendre le processus effectif, il faut une adéquation de l'infrastructure régionale, en améliorant les voies d'accès aux anneaux routiers, en diversifiant la structure d'évacuation de la production et en stimulant les activités substitutives, en plus de l'investissement en technologie et l'absorption du progrès technique, visant des gains de productivité et de qualité. Ces éléments croissent de manière importante au fur et à mesure que l'économie brésilienne vient se restructurer durant des années. La restructuration a été plus significative entre 1985 et 1995 . Pendant cette période, l'économie du Brésil est passée par de profondes transformations macroéconomiques, parmi lesquelles on compte la stabilisation des prix, l'ouverture commerciale, les changements dans la politique cambiste et l'entrée d'investissements externes directs.

En plus, le secteur de l'industrie agroalimentaire a eu une grande impulsion, principalement dans les régions Sud et Centre-Ouest. Les marques de cette impulsion ont été l'expansion de la production agro-pastorale dans le Cerrado, l'augmentation de la capacité installée des industries de viandes et de charcuterie dans l'État du Paraná et de Rio Grande do Sul et l'augmentation de la consommation de matières premières dans la chaîne productive de viande. De plus, l'augmentation des exportations pour le Moyen-Orient et l'Asie ont contribué à l'augmentation de la production et à l'occupation de la main-d'œuvre dans ces régions.

Cependant, la région Sud n'a pas été l'unique bénéficiaire de la restructuration spatiale de l'économie brésilienne. La région Sud-est a eu une avancée considérable dans la production de technologie de pointe, principalement en aérospatiale, en plus de continuer à être extrêmement dynamique par rapport aux autres régions. Ainsi, la déconcentration de la région métropolitaine de São Paulo n'a pas signifié une délocalisation de l'industrie de São Paulo, mais le transfert des activités de transformation dans 
des sous-régions localisées dans le propre État ou dans sa périphérie. D'une certaine forme, les nouveaux investissements dans le Paraná, principalement au Nord de l'État, au Sud-est de Minas Gerais et sur le littoral fluminense (de Rio de Janeiro) reflètent cette tendance.

Quant à elles, les régions Nord et Nord-est du Brésil ont connu un essor important de la production de biens manufacturés qui utilisent une main-d'œuvre peu qualifiée, parmi lesquelles on compte la production textile, de chaussures, l'extraction de minerais et l'expansion des activités touristiques. Les investissements dans le complexe automobile et pétrochimique dans la région de Bahia sont à souligner. Cependant, ces régions ont encore beaucoup de défis à venir, parmi lesquels on compte la récupération et l'amplification de l'infrastructure disponible, l'amplification de la qualification de la main-d'œuvre, des améliorations plus profondes dans les indicateurs sociaux, une amplification de la zone cultivable, etc. De toute manière, ces régions démontrent une tendance à la croissance économique ces dernières années, malgré les difficultés qu'elles affrontent en termes d'investissements et d'amélioration de la qualité de vie.

\section{Les Régions Nord et Nord-est du Brésil, elles ont connu un essor important de la production de biens manufacturés qui utilisent une main-d'œuvre peu qualifiée, parmi lesquelles on compte la production textile, de chaussures, l'extraction de minerais et l'expansion des activités touristiques. Les investissements dans le complexe automobile et pétrochimique dans la région de Bahia sont à souligner.}

\section{BIBLIOGRAPHIE}

${ }^{1}$ Krugman, P. (1991). «Increasing Returns and Economic Geography». Journal of Political Economy. no 99, p. 483-499.

${ }^{2}$ Ferrera De Lima, J. (2010). La diffusion spatiale du développement économique régional: l'analyse de la diffusion au sud du Brésil dans le $X X^{o}$ siècle. Sarrabruck: Éditions Universitaires Européennes.

Ferrera De Lima, J. (2012). Géoéconomie et développement régional . Paris: Publibook.
${ }^{3}$ Fonseca Netto, H. (2001). «Vers un nouveau découpage de l'espace brésilien». Revue Organisations et Territoires. Québec, n¹0, p. 99-110. 